\title{
Activated N-Ras signaling regulates arterial-venous specification in zebrafish
}

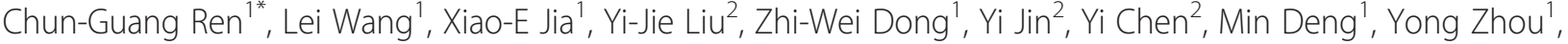 \\ Yi Zhou ${ }^{3}$, Rui-Bao Ren ${ }^{2 *}$, Wei-Jun Pan ${ }^{1 *}$ and Ting-Xi Liu' ${ }^{1,2}$
}

\begin{abstract}
Background: The aberrant activation of Ras signaling is associated with human diseases including hematological malignancies and vascular disorders. So far the pathological roles of activated Ras signaling in hematopoiesis and vasculogenesis are largely unknown.
\end{abstract}

Methods: A conditional Cre/loxP transgenic strategy was used to mediate the specific expression of a constitutively active form of human N-Ras in zebrafish endothelial and hematopoietic cells driven by the zebrafish Imo2 promoter. The expression of hematopoietic and endothelial marker genes was analyzed both via whole mount in situ hybridization (WISH) assay and real-time quantitative PCR (qPCR). The embryonic vascular morphogenesis was characterized both by living imaging and immunofluorescence on the sections with a confocal microscopy, and the number of endothelial cells in the embryos was quantified by flow cytometry. The functional analyses of the blood circulation were carried out by fluorescence microangiography assay and morpholino injection.

Results: In the activated N-Ras transgenic embryos, the primitive hematopoiesis appeared normal, however, the definitive hematopoiesis of these embryos was completely absent. Further analysis of endothelial cell markers confirmed that transcription of arterial marker ephrinB2 was significantly decreased and expression of venous marker flt4 excessively increased, indicating the activated N-Ras signaling promotes the venous development at the expense of arteriogenesis during zebrafish embryogenesis. The activated N-Ras-expressing embryos showed atrophic axial arteries and expansive axial veins, leading to no definitive hematopoietic stem cell formation, the blood circulation failure and subsequently embryonic lethality.

Conclusions: Our studies revealed for the first time that activated N-Ras signaling during the endothelial differentiation in vertebrates can disrupt the balance of arterial-venous specification, thus providing new insights into the pathogenesis of the congenital human vascular disease and tumorigenic angiogenesis.

Keywords: Vasculogenesis, Arteriogenesis, N-Ras

\section{Background}

RAS proteins are small GTPases that have been proved to be essential for the control of cell proliferation, survival, and differentiation [1,2]. Ras genes are evolutionally and functionally conserved in all eukaryotic organisms. In mammals, the 3 Ras genes encode 4 highly

\footnotetext{
* Correspondence: ren_chunguang@163.com; rbren@sjtu.edu.cn; weijunpan@sibs.ac.cn

'Key Laboratory of Stem Cell Biology, Institute of Health Sciences, Shanghai Institutes for Biological Sciences, Chinese Academy of Sciences \& Shanghai Jiao Tong University School of Medicine, Shanghai, China

${ }^{2}$ Shanghai Institute of Hematology, Ruilin Hospital, Shanghai Jiao Tong

University School of Medicine, Shanghai, China

Full list of author information is available at the end of the article
}

homologous proteins: $\mathrm{H}-, \mathrm{N}-$, and K-RAS 4A and 4B. The four RAS proteins interact with a common set of activators and effectors, and thus share many biochemical and biological functions [1]. Both Ras genes and components of Ras signaling pathways, including membrane receptor tyrosine kinases (RTKs), Ras-GEFs, Ras-GAPs, and downstream cytoplasmic kinases (RAFs), are reported to be frequently mutated in diverse human cancers and congenital developmental diseases [3]. In particular, activated mutations of N-Ras gene have been detected in human myeloid leukemias with high frequency [3], ranging from $15 \%$ to $60 \%$, and several myeloid leukemia-like mice models were established by

\section{Biomed Central}


inducing the expression of oncogenic N-Ras gene in bone marrow transduction/transplantation [4], transgenic [5] and knock-in [6,7] model systems, suggesting that the oncogenic N-Ras can initiate myeloid leukemias, nevertheless, the long latency and the requirement of cooperative genetic lesions in those models imply that the activated N-Ras signaling alone is insufficient to transform the normal hematopoietic cells into leukemic cells. So far the direct effect of oncogenic N-Ras signaling in hematopoietic cells remains mostly unknown.

Abnormal activation of Ras signaling has been reported to be involved in tumorigenic angiogenesis [8] and congenital vascular developmental diseases [3]. Capillary Malformation-Arteriovenous Malformation (CM-AVM) is a cutaneous congenital vascular disease that is compound of Capillary Malformation (CM) and Arteriovenous Malformation (AVM), and in which the arterial and venous vessels in the skin are connected directly to one another without an intervening capillary bed [9]. Rasa1 gene encodes p120ras-GAP which functions by inhibiting the activity of RAS proteins, and heterozygous inactivating rasa1 mutations were detected and proved to be the causal genetic lesion for CM-AVM [9], suggesting an important role of hyperactive Ras signaling in this disease. So far whether the rasa1 mutations function through an endothelial cell-autonomous manner is still unknown. In addition, the reciprocal signaling between EphrinB2 (artery specific gene) and its receptor EphB4 (vein specific gene) is critical for the formation of capillary beds [10], and it is proposed that a defect in ephrins or their receptors may be a causative factor in the formation of Arteriovenous Malformation (AVM) [11]. And notch pathway mutant (dll4 and Rbpsuh genes) mice embryos exhibit defects in arterial specification of nascent blood vessels and develop Arteriovenous Malformations [12]. However, the pathogenesis of CM-AVM or AVM is largely unknown.

In human N-RAS protein, a point mutation resulting in the substitution of glycine to aspartic acid at codon 12 (G12D) has been proved to impair GAP-stimulated GTP hydrolysis, making the N-Ras signaling constitutively activated [13], hereafter referred as hNRASD12. Lmo2 is a transcriptional factor specifically expressed in the hemangioblasts (the bipotential hemato-endothelial progenitor cells) and hemangioblasts-derived primitive hematopoietic and endothelial cells $[14,15]$. we recently established and functionally characterized the transgenic $\mathrm{Tg}$ (lmo2:Cre) fish expressing the Cre recombinase under the control of zebrafish $l m o 2$ promoter [16], and further showed that $\mathrm{Tg}(\operatorname{lmo} 2 \mathrm{Cre})$ mediated expression of dominant negative $\mathrm{C} / \mathrm{ebp} \alpha$ and $\mathrm{Bmi1}$ in zebrafish $\mathrm{lmo}^{+}$cells each extended short-lived hematopoietic stem/progenitor cell life span and induced lethal dyserythropoiesis [17]. To explore the roles of aberrant activation of
$\mathrm{N}$-Ras signaling in hematopoiesis and vasculogenesis, we used the $\operatorname{Tg}(\operatorname{lmo2}: \mathrm{Cr}$ ) to mediate the expression of $h N R A S D 12$ in $\mathrm{lmo}^{+}$cells of zebrafish embryos, and showed that the activated N-Ras signaling in the hNRASD12 transgenic embryos didn't induce overt hematopoietic or leukemic phenotypes, while caused severe defective vasculogenesis and subsequently embryonic lethality. The venous cell fate determination was abnormally enhanced at the expense of the arteriogenesis, indicating an endothelial cell-autonomous role of Ras signaling in the arterial-venous specification.

\section{Results}

Establishment of transgenic zebrafish lines with hemogenic and endothelial cell-specific expression of human oncogenic N-Ras

To address the roles of disease-associated activation of $\mathrm{N}$-Ras signaling in hematopoietic and endothelial systems in vivo, we induced specific expression of human NRASD12 (hNRASD12) in these tissues using the CreloxP system under the control of $l m o 2$ promoter in the zebrafish.

We first established the $T g(\beta$-actin:LDL-hNRASD12) lines (Figure 1A) and obtained 7 heterozygous stable lines that ubiquitously expressed the fluorescent DsRed protein during embryonic development (data not shown). All 7 lines were crossed to the heterozygous $\mathrm{Tg}$ (lmo2:Cre) fish individually to generate four different genotypes in the progeny: hNRASD12-Cre (wild-type), hNRASD12-Cre ${ }^{+}$, hNRASD12 ${ }^{+}$Cre ${ }^{-}(\beta$-actin:LDL-hNRASD12;wild-type) and hNRASD12 ${ }^{+} \mathrm{Cre}^{+}$( $\beta$-actin:LDL-hNRASD12; lmo2:Cre). In particular, the $\beta$-actin:LDL-hNRASD12;lmo2:Cre double transgenic embryos derived from line 4 showed robust expression of $h N R A S D 12$ transcripts in the intermediate cell mass (ICM) region at 22 hours post fertilization (hpf) (Figure 1B), which mostly resembled the expression pattern of endogenous $1 m o 2$ transcripts in wild-type embryos at the same stage (Figure 1D). And all the $\beta$-actin:LDLhNRASD12; wild-type embryos, hereafter referred as control embryos, showed no detectable expression of $h N R A S D 12$ transcripts in the ICM region (Figure $1 \mathrm{C}$ ). Consistently, the expression of hNRAS protein was only detected in the $\beta$-actin:LDL-hNRASD12;lmo2:Cre embryos at $28 \mathrm{hpf}$ (Figure 1E), hereafter referred as hNRASD12 embryos.

Elevated Ras signaling by overexpression of $h R A S D 12$ has no impact on primitive hematopoiesis

After confirmation of the $h N R A S D 12$ expression in the transgenic embryos, we examined the effect of activated $\mathrm{N}$-Ras signaling on the specification and differentiation of blood cells in the hNRASD12 embryos. Like mammalian, zebrafish hematopoiesis includes two consecutive waves of hematopoiesis: primitive and definitive 
A
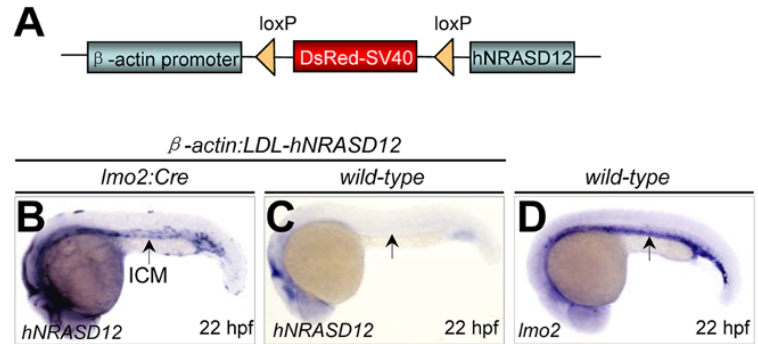

E

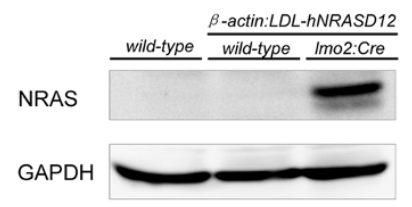

Figure 1 Validation of $h N R A S D 12$ expression in the $\beta$-actin:LDL-hNRASD12;Imo2:Cre transgenic embryos. (A) Diagrammatic scheme of the $\beta$-actin-LDL-hNRASD12 transgenic construct. (B-D) The expression analyses of the hNRASD12 transcripts via whole mount in situ hybridization (WISH) in the $\beta$-actin:LDL-hNRASD12;Imo2:Cre embryo (hereafter referred as hNRASD12 embryo) (B) and $\beta$-actin:LDL-hNRASD12; wild-type embryo (hereafter referred as control embryo) $(\mathbf{C})$ respectively at 22 hours post fertilization (hpf), and lom2 transcripts in the wild-type embryo at 22 hpf (D). Black arrows denote the location of the intermediate cell mass (ICM) region. (E) Confirmation of the expression of hNRAS protein in the hNRASD12-embryos at $28 \mathrm{hpf}$ via western blotting analysis.

hematopoiesis [18]. Primitive hematopoiesis originates from the anterior and posterior lateral plate mesoderm and produces erythrocytes, macrophages, and granulocytes, providing blood cells for early embryogenesis [18]. The definitive hematopoietic stem cells (HSCs) emerge from the hemogenic endothelium at the ventral wall of the dorsal aorta from $30 \mathrm{hpf}$ onward, called endothelial hematopoietic transition (EHT) [19,20], and generate all types of blood cells in adulthood [18]. To characterize the hematopoietic phenotypes in the hNRASD12 embryos, we performed whole mount in situ hybridization (WISH) analysis to examine the hematopoietic markers expression [21,22] in the control and hNRASD12 embryos at 18 and $22 \mathrm{hpf}$, including lmo2 (hemangioblasts), gata1 (primitive erythroid progenitors), ae1 globin (mature erythrocytes), pu.1 (primitive myeloid progenitors), l-plastin (primitive macrophages), mpo (neutrophils), cmyb and runx1 (HSCs). The results showed that expression of those primitive hematopoietic markers is not affected in the $h N R A S D 12$-expressing embryos (Figure 2A \& B). Consistent with the WISH results, the real-time quantitative PCR (qPCR) analysis showed no significant difference at the expression level of these markers between the $h N R A S D 12$ and control embryos,

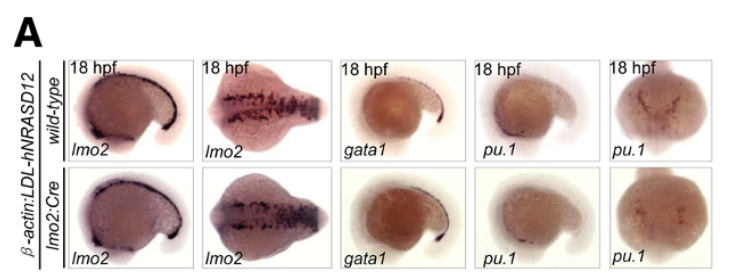

B

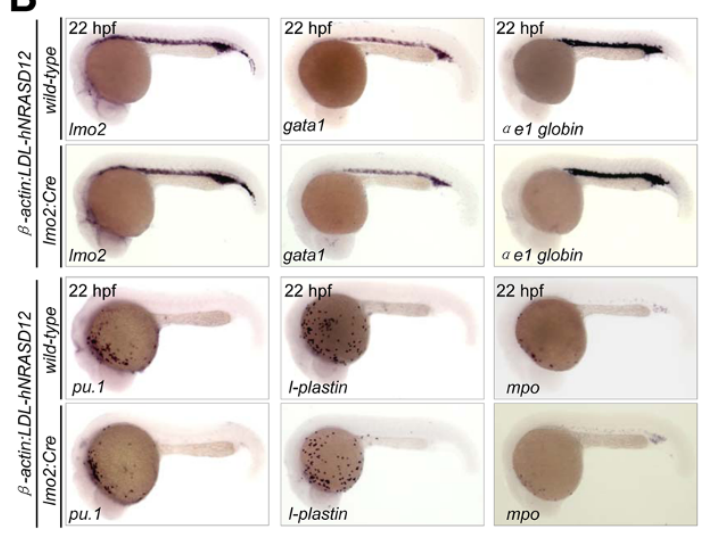

C

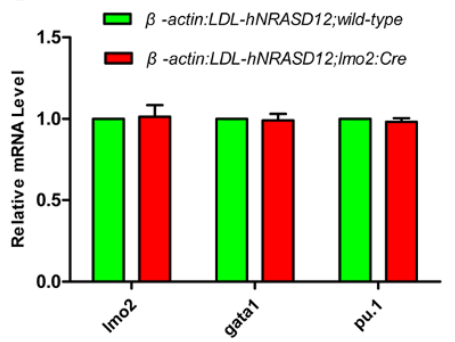

D

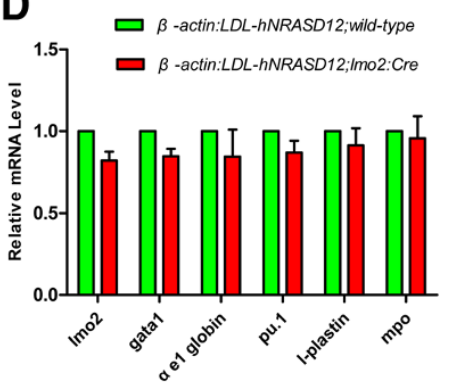

Figure 2 The primitive erythropoiesis and myelopoiesis are not affected by the expression of $h N R A S D 12$. (A-B) WISH analysis of primitive hematopoietic markers expression in the control embryos and hNRASD12expressing embryos respectively at 18 (A) and $22 \mathrm{hpf}$ (B). (C-D) Verification of the expression level of the hematopoietic markers in the control or hNRASD12 embryos at 18 (C) and 22 hpf (D) via real-time quantitative PCR. Data shown are means \pm SEM of at least three independent experiments. 
indicating the activated N-Ras doesn't alter the development of primitive blood cells (Figure 2C \& D). However, the definitive HSCs marked by runx1 and cmyb couldn't be detected in the aorta-gonad-mesonephros (AGM) region of the hNRASD12 embryos (Figure 3A-D). According to the defective blood circulation and dorsal aorta formation in the hNRASD12 embryos described below, the lack of definitive hematopoiesis is likely the secondary effect of the vascular defects, as both the normal blood circulation and dorsal aorta formation have been proven to be indispensable for the emergency of definitive HSCs in the AGM region [23,24], thus preventing from directly dissecting the role of activated N-Ras signaling in definitive hematopoiesis. Taken together, these data show that the oncogenic N-Ras signaling in the zebrafish $l m o 2^{+}$cells doesn't induce directly hematopoietic or leukemic phenotypes in the transgenic embryos.

\section{Vascular morphogenesis defects in hNRASD12-expressing transgenic embryos}

Expression of $h N R A S D 12$ under the $l m o 2$ promoter will lead to expression both in the hematopoietic and endothelial lineages, so we then examined the circulatory system and found the hNRASD12 embryos at $28 \mathrm{hpf}$ lacked blood circulation both in head and trunk vessels, and the blood cells were accumulated in the trunk axial vessels and heart chambers (Figure 4B \& D, and Additional files 1 and 2: movie S3 and S4) in comparison of normal

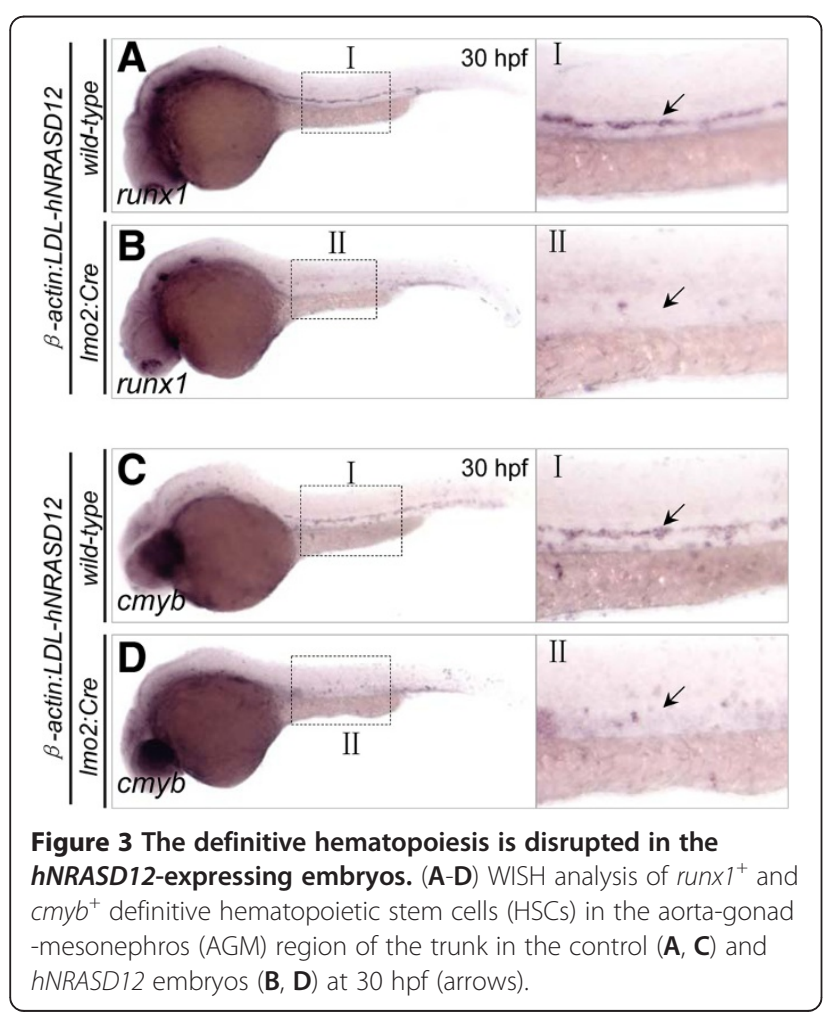

circulation in the control embryos (Figure 4A \& C, and Additional files 3 and 4: movie S1 and S2). The hNRASD12 embryos died between 5 and 8 days post fertilization (dpf) with the persistent lack of blood circulation. To validate the blockage of circulation was specifically induced by the expression of human NRASD12, we designed a rescue experiment by using an antisense ATG morpholino (MO) targeting the translational initiation site of this transgenic hNRASD12. Firstly, the efficiency of the hNRAS MO was confirmed by efficiently blocking both the translation of the GFP mRNA reporter (Additional file 5: Figure S1A-D) in wild-type embryos and the expression of hNRASD12 protein in the hNRASD12 embryos (Additional file 5: Figure S1E). Compared with the control MO, the hNRAS MO that was injected into one-cell stage embryos derived from the crossing between the heterozygous $\mathrm{Tg}(\operatorname{lmo2}: \mathrm{Cre})$ and $T g(\beta$-actin:LDL-hNRASD12) fish restored the blood circulation of $h N R A S D 12$ embryos to a comparable level as that of control embryos (Figure 4E), indicating the blocked blood circulation is specifically induced by $h N R A S D 12$ ectopic expression. Considering the primitive hematopoiesis of $h N R A S D 12$ embryos is normal, we hypothesized that the congestion of circulation resulted from the defective cardiovascular development. We then checked the cardiovascular structure and function by using the fluorescent microangiography assay. The fluorescein-coupled latex beads were injected into the inflow tract of the atrium of the control and $h N R A S D 12$ embryos respectively, and were observed to perfuse the whole vascular system of the control embryos in five minutes (Additional file 5: Figure S2A, upper panel). However, it took over 30 minutes for injected beads to passively diffuse into the anterior trunk of the $h N R A S D 12$ embryos (Additional file 5: Figure S2A, lower panel), indicating the activated Ras signaling disrupts the circulation. In particular, the consecutive accumulation of fluorescent beads in the trunk of hNRASD12 embryos (Additional file 5: Figure S2A, lower panel) suggested the existence of only one axial vascular tube. We further examined the function of the heart of hNRASD12 embryo by counting the rate of heartbeats, and the result showed that the average rate of heartbeats in the hNRASD12 embryos at $30 \mathrm{hpf}$ was only slightly slower than that of control embryos (Additional file 5: Figure S2B), suggesting the phenotype of heartbeat contributes little to the circulation blockage.

To further analyze the vascular defect in the hNRASD12 embryos, we crossed the $T g(f l k 1: G F P)$ fish [25], an endothelial reporter transgenic line, with $\operatorname{Tg}(\beta$-actin: $L D L$ $h N R A S D 12)$ fish to obtain the double transgenic progeny, which were raised to adulthood and subsequently crossed to $\mathrm{Tg}$ (lmo2:Cre) fish to generate the $\beta$-actin:LDLhNRASD12;flk1:GFP; lmo2:Cre (referred as hNRASD12; $f l k 1: G F P)$ embryos. In the trunk of $\beta$-actin:LDL- 

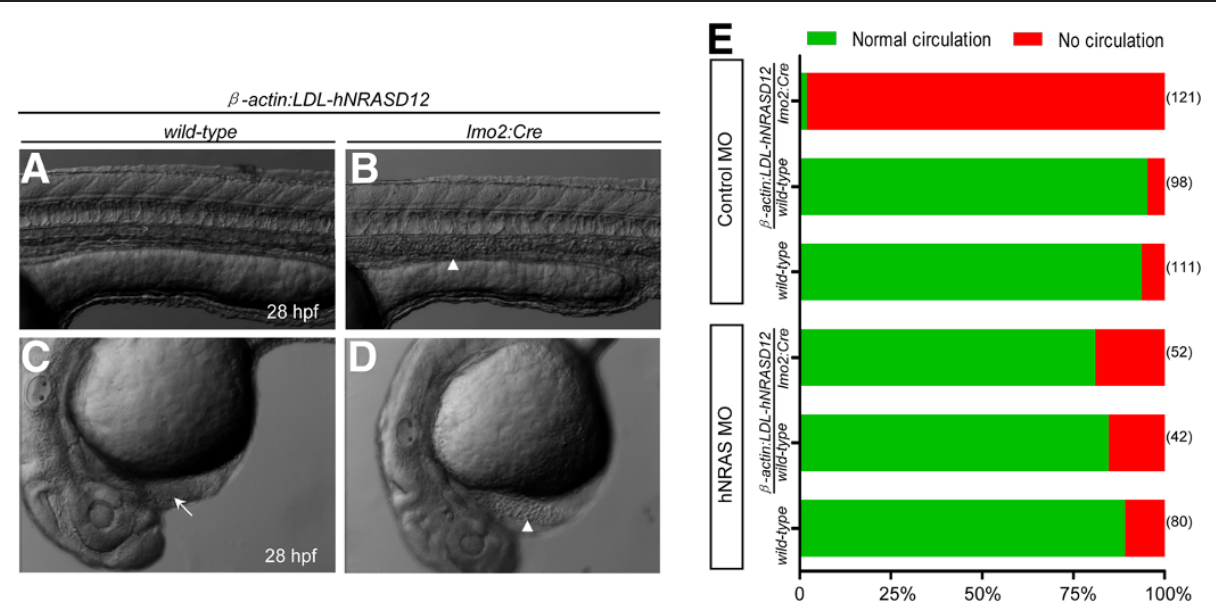

Figure 4 The expression of $h N R A S D 12$ blocks the blood circulation. (A-D) Morphology of the control (A \& C) and hNRASD12 embryos $(\mathbf{B} \& \mathbf{D})$ at $28 \mathrm{hpf}$. The arrows mark the blood flow in the trunk and heart of control embryo, and the triangles denote the accumulated blood cells in the trunk and heart of hNRASD12 embryo. (E) The assessment of blood circulation in wild-type, control and hNRASD12 embryos after injection of control and hNRAS morpholino $(\mathrm{MO})$ respectively; the numbers on the right of the bars indicate the amount of the embryos being counted, and representative results obtained from three independent experiments.

hNRASD12;flk1:GFP;wild-type (referred as control;flk1: GFP) embryos at $28 \mathrm{hpf}$, the primary blood vessels consisted of the dorsal aorta (DA) and posterior cardinal vein (PCV), and the intersegmental vessels (ISVs) formed by sprouting from the dorsal aorta (Figure 5A) [26]. However, hNRASD12;flk1:GFP embryos failed to form morphologically distinct DA or PCV, and the ISVs were also defective (Figure 5B). The results of immunofluorescence analysis on transverse sections showed the presence of only one vascular tube in the trunk of $h N R A S D 12-$ expressing embryo (Figure 5C \& D). Furthermore, the morphology of head vasculature was also examined, in the control;flk1:GFP embryos at $28 \mathrm{hpf}$, the paired bilateral lateral dorsal aortas (LDAs) merged backward into single dorsal aorta at the boundary between head and trunk, and the posterior cardinal vein (PCV) split forward into a pair of vessels in the cranial trunk, then the branches of $\mathrm{PCV}$ emptied into the common cardinal veins (CCVs) on the either side of yolk, which came into the sinus venous of the heart (Figure 5E \& G) [26]. Unlike that of control;flk1: GFP embryos, the LDAs of hNRASD12;flk1:GFP embryos were evidently atrophic while CCVs and the bifurcation of PCV were greatly expanded, however, the primordial hindbrain channels (PHBCs), the primary veins in the head at this stage, seemed normal (Figure 5F \& H). In addition, basilar artery (BA), the main artery in the hindbrain of the zebrafish embryo formed by endothelial sprouting of PHBCs [27], was lost in the hNRASD12-expressing embryo (Figure 5I \& J). Taken together, our observations suggested that the activated N-Ras signaling in the zebrafish $l \mathrm{mo}^{+}$cells caused the abnormal assembly of the axial blood vessels and the defective arteries formation and expansion of veins in the head vasculature system of the $h N R A S D 12$ embryos.

\section{hNRASD12 promotes venous fate specification at the expense of arterial fate in zebrafish}

As Ras signaling has been proved to be important for cell proliferation $[1,2]$, the frequencies of endothelial cells in the hNRASD12;flk1:GFP and control;flk1:GFP embryos at $28 \mathrm{hpf}$ were respectively quantified by flow cytometry analysis, and the result showed no significant difference in the frequencies of $\mathrm{GFP}^{+}$endothelial cells between hNRASD12-expressing and control embryos (Figure 6A). We further characterized the arterial and venous cells lineages respectively in the $h N R A S D 12 \mathrm{em}-$ bryos by performing WISH analysis. EphrinB2, hRT, dll4 and $g r l$ genes are specifically expressed in the arterial cells of wild-type embryo's trunk region [23,27], while ephB4, flt4 and dab2 mark the venous cells [28]. The result of WISH showed that the arterial cells were evidently reduced in $h N R A S D 12-$ expressing embryos compared with control embryos (Figure 6B, B', D, and D'), and failed to form an intact dorsal aorta (Figure 6C, C, $\mathrm{E}$, and $\left.\mathrm{E}^{\prime}\right) ;$ On the contrary, the venous cells in the hNRASD12 embryos were greatly expanded and organized to be an enlarged vein-like vascular tube (Figure 6F-I and F'-I'). To confirm that expression of hNRASD12 induced an unbalanced arterial-venous specification in the same embryo, we performed 2-color WISH analysis with digoxigenin-labeled ephrinB2 and fluorescein-labeled flt4 antisense probes, and observed the expansion of venous cells and reduction of arterial cells occurring in a single hNRASD12-expressing embryo (Figure 6)' \& K') compared 

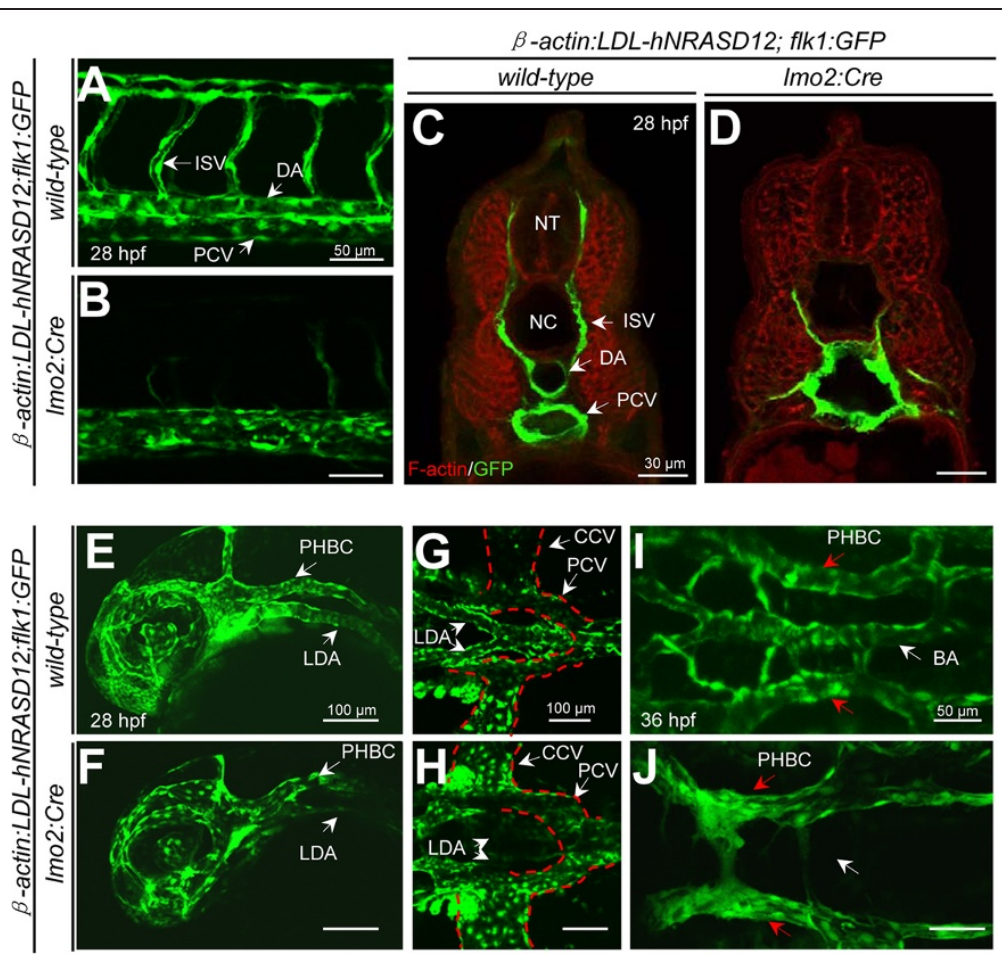

Figure 5 Vasculogenesis is disrupted in hNRASD12-expressing transgenic embryos. (A-B) Confocal images of trunk vasculature of living control; flk1:GFP (A) and hNRASD12;flk1:GFP embryos (B) at 28 hpf. DA, dorsal aorta; PCV, posterior cardinal vein; ISV, intersegmental vessel. (C-D) Mid-trunk transverse sections of control;flk1:GFP (C) and hNRASD12;flk1:GFP embryos (D) at 28 hpf stained by GFP antibody (green) and PhalloidinTRITC (red). NT, neural tube; NC, notochord. (E-J) Confocal images of head vasculature of living control;flk1:GFP (E, G, I) and hNRASD12;flk1:GFP embryos at $28 \mathrm{hpf}(\mathbf{F}, \mathbf{H}, \mathbf{J})$. LDA, lateral dorsal aorta; PHBC, primordial hindbrain channel; CCV, common cardinal vein; BA, basilar artery. The red dotted lines denote the boundaries of the CCVs and PCVs respectively.

to that in control embryo (Figure 6J \& K). These results from WISH with dll4, grl, and dab2 probes also supported the above observations (Additional file 5: Figure S3 A-F) that almost the entire vascular tube in the trunk of hNRASD12 embryo consists of venous endothelial cells.

Vasculogenesis is referred to as the de novo formation of the blood vessels, involving the differentiation, proliferation and migration of endothelial cells [29]. In zebrafish, the angioblasts at the bilateral posterior lateral plate mesoderm migrate medially to form the vascular cord between 14 and $18 \mathrm{hpf}$, meanwhile the arterialvenous specification is established $[23,25]$. Then the venous cells sprout ventrally to separate from the arterial cells between 21 and $24 \mathrm{hpf}$, called arterial-venous segregation [30]. Subsequently, the dorsal arterial cells organize to be dorsal aorta by cord hollowing [31], while the posterior cardinal vein forms by embrace of the hematopoietic cells [30]. To figure out which of the above developmental processes is affected firstly by the expression of $h N R A S D 12$, we used WISH analysis to detect the arterial-venous specification at earlier stages before $28 \mathrm{hpf}$, and found that arterial-venous defects in the hNRASD12-expressing embryos appeared as early as 20 hpf (Figure 7A-L), when the identity of arterial-venous cell fate is just established [25], indicating N-Ras signaling regulates the specification of arterial and venous cells before the arterial-venous segregation and onset of circulation. In addition, previous studies have revealed an important role of the angioblasts migration between 14 and $18 \mathrm{hpf}$ in the arterial-venous specification [23,32]. To examine whether the constitutively activated N-Ras signaling affects the medial migration of angioblasts, we used $f l k 1$ as a probe to trace this process. The result showed the medial migration began at 14-15 hpf, and nearly all angioblasts arrived at the midline at $17-18 \mathrm{hpf}$ in the control embryo (Figure 8A-E), and hNRASD12-expressing embryo showed a similar pattern (Figure 8F-J), indicating the activated N-Ras signaling specifically affects the arterial-venous specification in an angioblasts-medialmigration-independent manner.

To further confirm that the defects in the assembly of the blood vessels and the arterial and venous fate decisions are specifically induced by the transgenic expression of hNRASD12, we knocked down the hNRASD12 expression using hNRAS MO in the hNRASD12 embryos. The trunk vasculature was imaged under a confocal microscopy and arterial and venous cells in the region were evaluated by WISH analysis of arterial- 

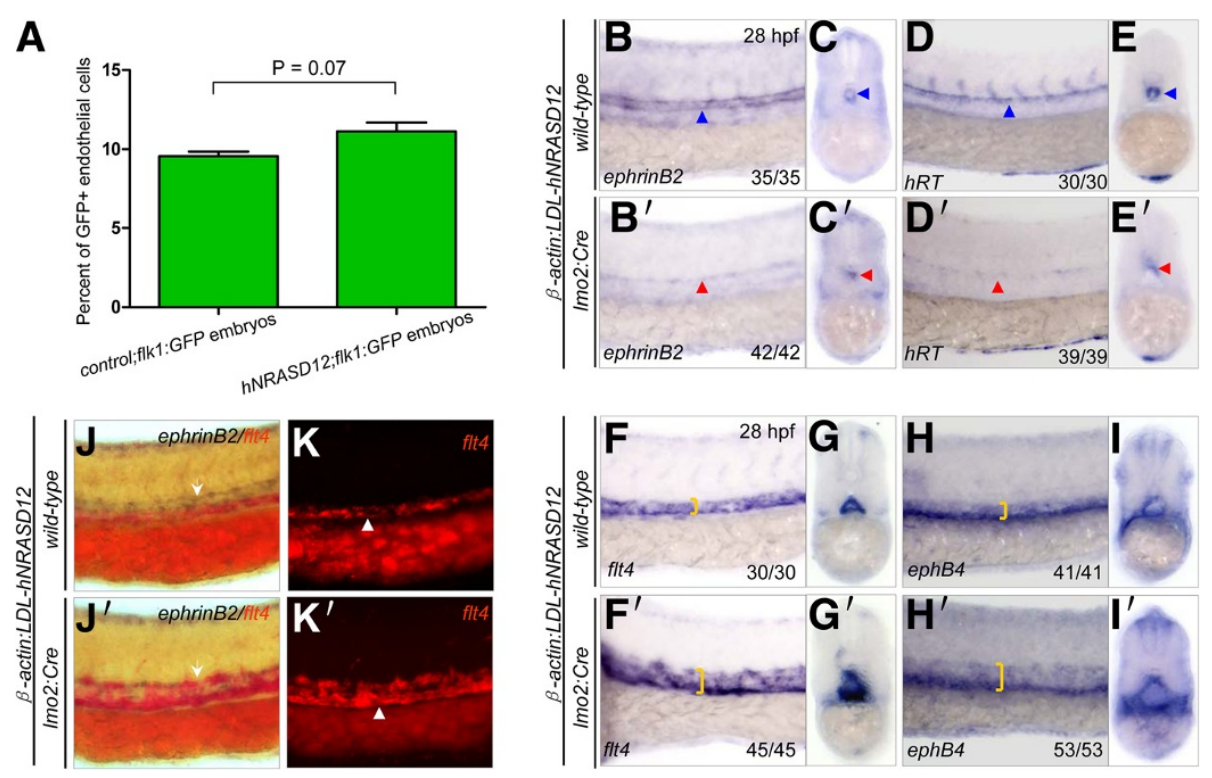

Figure 6 Expression of $h N R A S D 12$ promotes venous specification at the expense of arterial fate during embryonic endothelial differentiation. (A) Quantitation of total endothelial cells by flow cytometry analysis on the control;flk1:GFP and hNRASD12;flk1:GFP embryos at 28 hpf. Representative results obtained from three independent experiments. 2 tailed $t$-test of frequency of endothelial cells in hNRASD12;flk1:GFP embryos vs control;flk1:GFP embryos shows no significant difference. (B-E') WISH analysis of ephrinB2 ${ }^{+}$and $h R T^{+}$arterial cells in the trunk of control

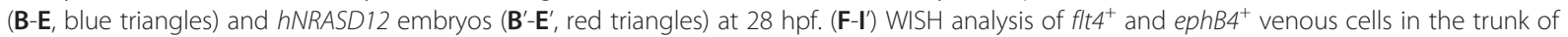
control (F-I) and hNRASD12 embryos ( $\left.\mathbf{F}^{\prime}-\mathbf{I}^{\prime}\right)$ at 28 hpf. The yellow square brackets mark the width of the venous strip in the trunk. (J-K') 2-color WISH analysis with digoxigenin-labeled ephrinB2 (white arrows) and fluorescein-labeled flt4 (white triangles) in control (J, K) and hNRASD12 embryos $\left(\mathbf{J}^{\prime}, \mathbf{K}^{\prime}\right)$ respectively at $28 \mathrm{hpf}$.

venous cells specific markers. Compared to the control MO, the hNRAS MO efficiently rescued both the defective assembly of blood vessels (Additional file 5: Figure S4A) and the unbalanced arterial-venous fates of the endothelial cells (Additional file 5: Figure S4B) in the trunk region of $h N R A S D 12$ embryo, indicating the defective vasculogenesis in the transgenic embryos is specifically caused by $h N R A S D 12$ expression.

Thus we conclude that the activated N-Ras signaling by expressing $h N R A S D 12$ in $l m o 2^{+}$cells disrupts the balance of the arterial-venous specification, where the venous fate decision is enhanced while the arterial specification is repressed, which in turn causes abnormal assembly of main blood vessels and the defect in the development of intersegmental vessels, leading to blockage of blood circulation and embryonic lethality. Nevertheless, expression of $h N R A S D 12$ affects neither the endothelial specification from mesoderm cells, the medial migration of angioblasts, nor the proliferation of endothelial cells.

The hierarchical signaling molecules, including sonic hedgehog (Shh), vascular endothelial growth factor A (VEGFA), phospholipase C gamma-1 (Plcg1), and notch have been shown to be essential for the arterial cell fate decision [33]; while chicken ovalbumin upstream promoter-transcription factor II (COUP-TFII), also known as Nuclear Receptor 2F2 or NR2F2, is indispensable for the venous specification [33]. To figure out whether hNRASD12 regulates the transcriptional expression of those genes, we performed WISH analysis with riboprobes of these arterial-venous regulatory genes, and found no significant difference between the control and hNRASD12 embryos (Additional file 5: Figure S5A-F and S5A'-F').

Previous studies have shown that forced expression of vegfaa ${ }_{121}$ in wild-type embryo could transform all the endothelial cells into ephrinB2 $2^{+}$arterial cells [34], however, we observed the failure of $h N R A S D 12$ embryos to respond to exogenous expression of vegfaa 121 (Additional file 5: Figure S6A-E), indicating that N-Ras signaling functions downstream of vegfa to negatively regulate the vegfa induced arterial development.

Considering notch signaling has been proved to act downstream of shh-vegfa signaling axis to regulate the arterial endothelial differentiation in zebrafish [35], we tried to rescue the hNRASD12-induced defective arteriogenesis by injecting a transient transgenic construct into the hNRASD12 embryos, in which a constitutively activated form of notch1 containing only the intracellular domain (referred as NICD1) was driven by the zebrafish flk1 promoter, however, no recovery of arteriogenesis was observed in those injected embryos (data not shown), implying defects caused by activated Ras is not through disrupting the notch signaling. 

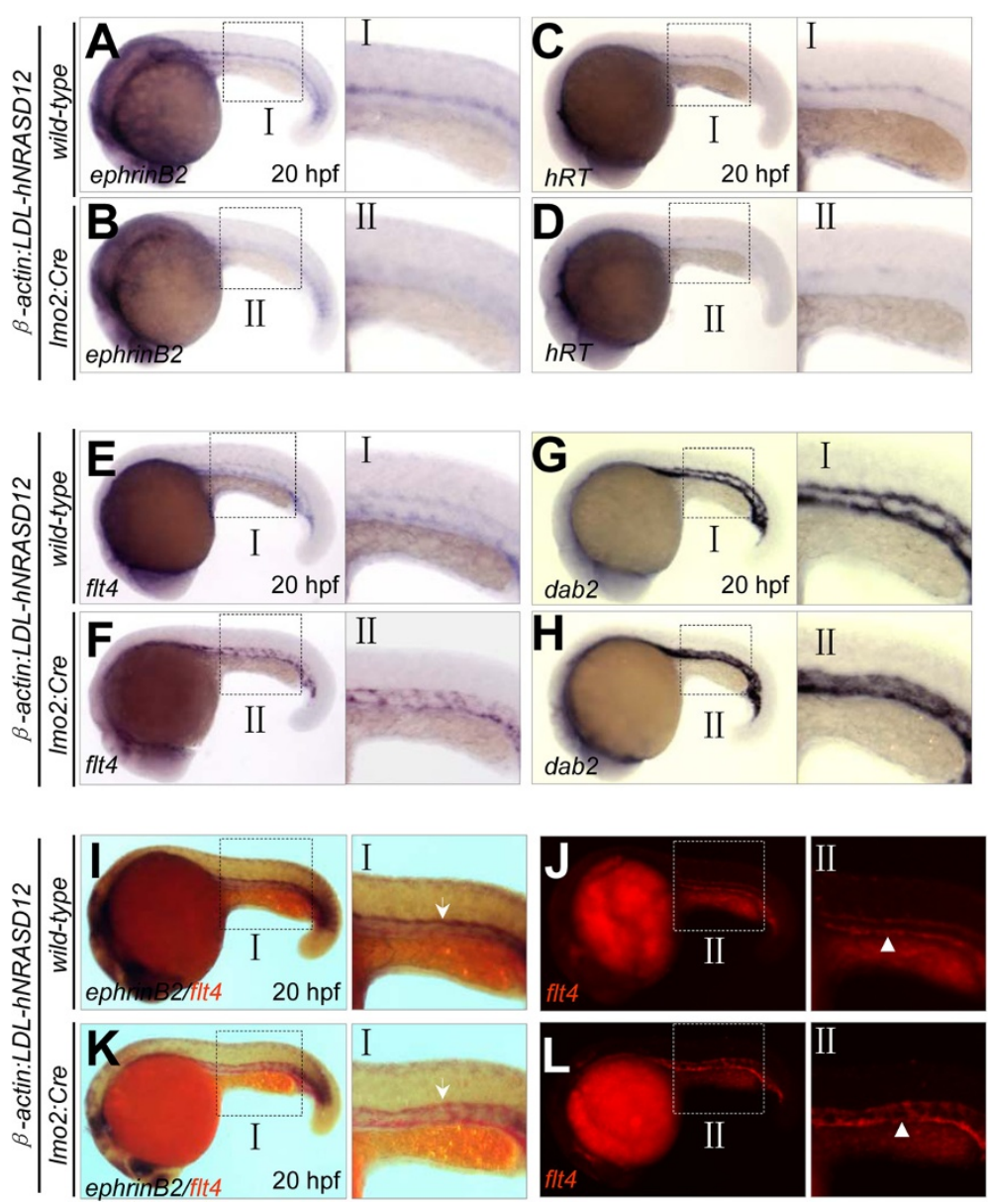

Figure 7 Loss of arterial specification by $h N R A S D 12$ expression before arterial-venous segregation and onset of circulation. (A-H) WISH

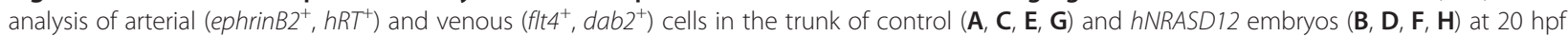
respectively. (I-L) 2-color WISH analysis with digoxigenin-labeled ephrinB2 (white arrows) and fluorescein-labeled flt4 (white triangles) in control (I, J) and hNRASD12 embryos (K, L)

Functional conservation of human and zebrafish N-Ras signaling in vasculogenesis

To assess the conservation between the human and zebrafish N-Ras signaling, we compared the protein sequences of the human, mouse and zebrafish N-RAS, and found that there is a high degree of sequence similarity between the zebrafish and mammalian N-RAS with a high identity of 95\% in the critical H_N_K_Ras_like domain (G domain) (Additional file 5: Figure S7). To further determine whether the activated zebrafish N-Ras signaling has a conserved function in the vasculogenesis as human N-Ras, we generated both lmo2:EGFP-2AhNRASD12 and lmo2:EGFP-2A-zNRASD12 transgenic constructs, in which the zebrafish $l m o 2$ promoter was used to transiently direct the specific expression of activating mutant of human and zebrafish N-Ras in blood and vasculature respectively. The WISH analysis showed that both the hNRASD12 and zNRASD12 transcripts were appropriately expressed in the ICM region
(Additional file 5: Figure S8A), similar to the expression pattern of endogenous $l m o 2$ gene (Figure 1D), and the expression of the human and zebrafish NRASD12 proteins was also confirmed via western blotting assay (Additional file 5: Figure S8B). The trunk vasculature of embryos expressing the hNRASD12 or $z N R A S D 12$ was characterized under a confocal microscopy. And compared to the embryos injected with lmo2:EGFP$2 A$, both the hNRASD12 and $z N R A S D 12$-expressing embryos had only one vascular tube in the trunk (Additional file 5: Figure S8C). Additional WISH analysis of the arterial marker ephrinB2 and venous marker flt4 genes showed large expansion of venous cells population and nearly lack of the arterial cells in the trunk region (Additional file 5: Figure S8C). These results strongly suggest a conserved function between activated human and zebrafish N-RAS and that the $\mathrm{N}$-Ras signaling participate in regulating the zebrafish vascular development. 


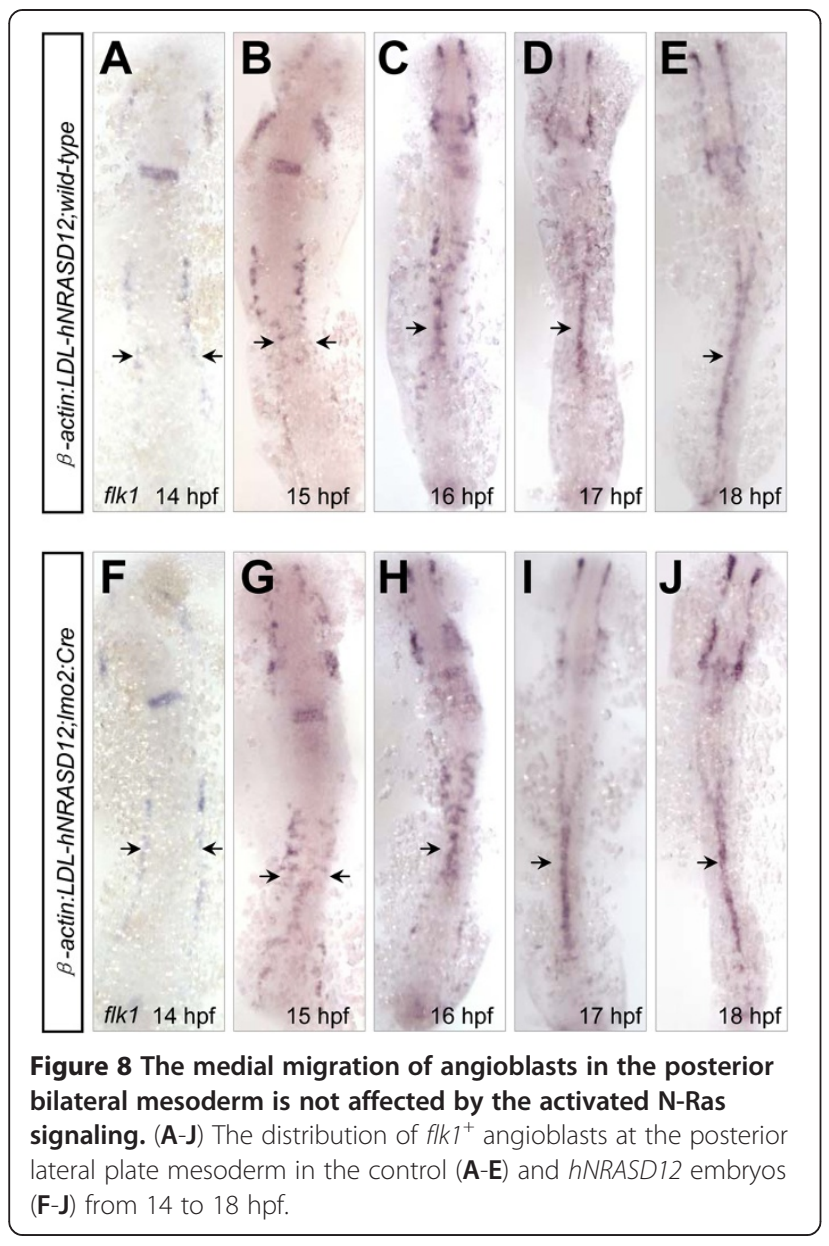

\section{Discussion}

In this study, we showed that transgenic overexpression of activated human NRAS (hNRASD12) in zebrafish $l m o 2^{+}$cells caused un-circulated vasculature, but had no impact on the primitive hematopoiesis during embryogenesis. In particular, the expression of $h N R A S D 12$ promoted the venous fate at the expense of arterial fate during arterial-venous specification, indicating an important novel role of activated Ras signaling in vascular morphogenesis.

Our whole mount in situ hybridization (WISH) and quantitative RT-PCR analysis of the expression of the hematopoietic markers showed the expression of hNRASD12 in the $l m o 2^{+}$hemangioblasts neither affected the specification or differentiation of the primitive hematopoietic cells, nor induced any leukemic phenotypes. However, the mice models expressing hNRASD12 exhibited leukocytosis and progressed to myeloid leukemias [4-7]. The discrepancy between our zebrafish model and those mice models is likely due to the difference in the targeting cell type and the developmental window of the NRASD12 expression. In the mice models, the expression of the NRASD12 was induced in the adult bone marrow cells, the adult hematopoietic organs, and caused dysplastic myelopoiesis/leukemias with long latencies $(>4$ weeks), which highlight the possible requirement of a definitive hematopoietic environment and the length of time to accumulate cooperative genetic mutations for the development of NRASD12-associated leukemia [36]. In our study, the expression of $h N R A S D 12$ was activated during the transient primitive hematopoietic stage and embryos failed to develop through the definitive hematopoietic stages due to lack of definitive hematopoiesis as a result of the defective vasculogenesis, and early embryonic lethality (5-8 days post fertilization), preventing the occurrence of the myeloid disorders and leukemias. Our results supported the concept that leukemogenesis is a multistep process involving cooperating genetic mutations [37], the elevation of RAS signaling by the expression of $h N R A S D 12$ alone in the short-lived primitive hematopoietic cells was insufficient for leukemogenesis.

Whether the hyperactive Ras signaling caused by inactivating RASA1 mutations in Capillary Malformation-Arteriovenous Malformation (CM-AVM) functions through an endothelial cell-autonomous manner is still unknown [9]. Here we showed that the expression of oncogenic human $\mathrm{N}$-Ras in the $l m o 2^{+}$cells disrupted arterial-venous specification and angiogenesis in zebrafish embryos, indicating an endothelial cell-autonomous role of oncogenic ras signaling in vasculogenesis. Consistently, the rasa1-deficient mice embryos are embryonic lethal and exhibit atrophic dorsal aorta and disorganized patterns of the intersegmental arteries [38]. The EphrinB2/ EphB4 signaling [10,11] and notch pathway [12] are involved in the pathogenesis of Arteriovenous Malformation (AVM), suggesting the direct genetic connection between the arterial-venous specification and CM-AVM/AVM. In our study, the transgenic expression of hNRASD12 induces defective arterial-venous specification in zebrafish, and this vertebrate genetic model may help us to further dissect the pathogenesis of CM-AVM or AVM.

In mice, it has been demonstrated that the germ line oncogenic Ras expression leads to embryonic lethality [6,39], where the Protamine-Cre (PrmCre) mice mediates constitutive and ubiquitous expression of oncogenic KRASD12 (floxed knock-in) [39], and mox2-Cre mice mediates germ line expression of endogenous oncogenic NRASD12 [6], however, the cellular and pathological mechanisms for the embryonic lethality in those studies are unknown. Here we show that the expression of oncogenic hNRASD12 during zebrafish embryogenesis induces embryonic lethality through disrupting the arterial-venous cell fate decision, offering a possible mechanistic explanation for the oncogenic Ras signaling induced embryonic lethality. Nevertheless, the phenotypes of endothelial development need to be further examined in those mice models [6,39]. 
The zebrafish has emerged as the first vertebrate model organism that is suitable for large-scaled wholeanimal small chemical molecule screening, contributing to several aspects of the drug development process, including target identification, disease modeling, lead discovery and toxicology [40]. The oncogenic NRASD12expressing mice develop leukemias in adult stage [4-7], and are not suitable for drug screening due to the cost and the large size of an individual, while the NRASD12 transgenic zebrafish embryos may be helpful for us to perform large-scaled whole-animal small chemical molecule screening to find oncogenic NRASD12-associated modulators using a developmental stage-controlled expression of oncogenic NRASD12.

\section{Conclusions}

In conclusion, we report firstly the constitutively activated N-Ras signaling regulates the arterial-venous specification in vertebrate, and also established an oncogenic $h N R A S D 12$ induced animal disease model that is feasible for high-throughput whole-animal drug screening and validating the functionality of NRASD12 modulators as potential therapeutics for a variety of human diseases caused by oncogenic mutant RAS.

\section{Methods}

Fish care

Zebrafish maintenance, breeding, and staging were performed as previously described [41]. The zebrafish experiments were approved by the Animal Experimentation Committee of Shanghai Institutes for Biological Sciences and institutional review board of Institute of Health Sciences.

\section{Generation of $T g(\beta$-actin:LDL-hNRASD12) transgenic line}

Human NRASD12 (hNRASD12) was obtained by PCR from a myc-tagged NRASD12 in a retroviral vector [4] and cloned into pCS2+ vector at the ClaI and $\mathrm{XhoI}$ sites to get the hNRASD12-pCS2+ construct. Then the $h N R A S D 12-S V 40$ fragment was excised from $h N R A S D 12-p C S 2+$ plasmid by $\mathrm{ClaI}$ and $\mathrm{KpnI}$ and cloned into the same sites of $\beta$-actin-loxP-DsRed-SV4OloxP-PBSK-I-SceI plasmid [16] to get the $\beta$-actin-loxPDsRed-SV40-loxP-hNRASD12-PBSK-I-SceI transgenic construct. Then this construct was co-injected with I-SceI meganuclease (New England Biolabs) into the fertilized zebrafish eggs at one cell stage. The injected embryos were raised to adulthood and crossed to wild-type fish to generate the F1 progeny, and the transgenic founders were identified by screening for the F1 embryos, expressing DsRed fluorescence at 24 hours post fertilization (hpf). We raised the DsRed ${ }^{+} \mathrm{F} 1$ embryos to adulthood to establish the stable transgenic lines.

\section{Transient transgenic constructs}

The zebrafish $l m o 2$ promoter of $2.5 \mathrm{kbp}$ was obtained by PCR amplification from the lmo2-Cre-PBSK-I-SceI plasmid [16] and cloned into the EGFP-2A-pDestTol2 plasmid (Z.W.Dong et al., unpublished data) at the XhoI and BamHI sites to get the lmo2-EGFP-2A-pDestTol2 construct. Then the human NRASD12 (hNRASD12) and zebrafish NRASD12 (zNRASD12) fragments were obtained by PCR from the $\beta$-actin-loxP-DsRed-SV40-loxPhNRASD12-PBSK-I-SceI plasmid and CDNA from the wild-type embryos respectively, and cloned into the $l m o 2-$ EGFP-2A-pDestTol2 construct at the XmaI and SalI sites to get the Imo2-EGFP-2A-hNRASD12/zNRASD12pDestTol 2 constructs. Of note, the forward primer for the amplification of zebrafish NRASD12 contains mutations at the codon 12 from GGA to GAT, resulting in the substitution of glycine $(G)$ to aspartic acid (D). Then the lmo2-EGFP-2A-pDestTol2, lmo2-EGFP-2A-hNRASD12pDestTol2 and lmo2-EGFP-2A-zNRASD12- pDestTol2 transgenic plasmids (25 ng/ul) were individually coinjected with $\mathrm{KCl}(0.2 \mathrm{M})$ and Tol2 transposase mRNA (25 ng/ul) as previously described [42] into the $\operatorname{Tg}(f l k 1$ : $m$ Cherry) or wild-type zebrafish embryos at one cell stage.

To generate the construct of the zebrafish $f l k 1$ promoter driven NICD1, the HA-tagged NICD1 fragment was obtained by PCR from the PME-NICD1 (zebrafish) plasmid and cloned into the flk1-PolyA-pBSKI2 plasmid at the BamHI and EcoRI sites to get the $f l k 1-H A$ NICD1-PolyA-pBSKI2 construct. Then the construct was co-injected with I-SceI meganuclease into the hNRASD12 transgenic embryos at one cell stage.

\section{Morpholino (MO)}

Embryos were injected with 2 nl human NRAS ATG MO (Gene Tools): 5'-ACCAGTTTGTACTCAGTCATAT CGA-3'. The efficiency of hNRAS MO was confirmed both by western blotting and co-injection with $h N R A S^{1-60}$ GFP mRNA reporter containing the targeting sites of hNRAS MO.

\section{Preparation of mRNAs and antisense probes, whole- mount in situ hybridization (WISH)}

A 60 bp fragment of human NRAS that contains the hNRAS ATG MO binding sites was fused to the $\mathrm{N}$ terminal of GFP in the pCS2+ vector to get the $h N R A S^{1-60}$ GFP-pCS2+ construct. The hNRAS $S^{1-60}-G F P$, zebrafish vegfaa $_{121}$ [35] and Tol2 transposase [43] mRNAs were synthesized with mMESSAGE mMACHINE Kit (Ambion).

The digoxigenin- or fluorescein-labelled antisense probes used for WISH analysis in this study includes lmo2 [16], (shh, notch1) [17], vegfaa 121 [35], (plcg1, pu.1,

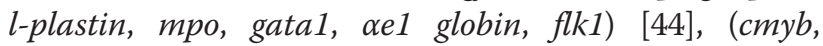
runx1) [45], (ephrinB2, hRT, grl, notch3) [23], (dab2, ephB4 and flt4) [28]. And the hNRASD12, zNRASD12, 
dll4 and $n r 2 f 2$ were cloned into $\mathrm{pCS} 2+$ plasmid and their digoxigenin-labelled antisense probes were synthesized with T3 polymerase (Ambion).

Single- and 2-color WISH were performed as described previously [17]. Embryos were mounted in 3\% methylcellulose and captured under the Nikon SMZ1500 microscope equipped with a Nikon DXM1200F digital camera and ACT-1 software.

\section{Genomic DNA isolation and genotyping}

Embryos were incubated in lysis buffer (Tris-HCl $1 \mathrm{M}$, pH 8.3; KCl 1 M; Tween 20 10\%; NP40 10\%) and protease $\mathrm{K}(10 \mathrm{mg} / \mathrm{ml})$ at $55^{\circ} \mathrm{C}$ overnight. Then $3700 \mathrm{rpm}$ for $10 \mathrm{~min}$ at $4^{\circ} \mathrm{C}$, and the supernatant was subject to genomic PCR with specific primers for Cre and hNRASD12.

\section{Real-time Quantitative PCR}

Total RNAs were extracted from 20 zebrafish embryos using Trizol reagent (Invitrogen). RNA was reversetranscribed using random hexamers and SuperScript III Reverse Transcriptase (Invitrogen). 2×PCR Mix (TaKaRa, Premix Ex $\mathrm{Taq}^{\mathrm{TM}}$ ) containing SYBR Green I was used for the real-time quantitative PCR analysis with the Applied Biosystems 7900HT Fast Real-Time PCR System. The relative expression values were normalized against the internal control gapdh (qPCR primer sequences were listed in Additional file 5: Table S1).

\section{Western blotting and immunofluorescence}

The western blotting assay was performed as described previously [17] with antibodies: human NRAS (Santa Cruz), GAPDH (Cell Signaling Technology). Immunohistochemistry was performed as described previously [25], and the trunk of embryo was transversely sectioned with a Leica VT1000S vibratome into 50-100 $\mu \mathrm{m}$ sections, and F-actin was stained with Phalloidin-TRITC (0.007 $\mu \mathrm{M}$, Sigma), and GFP antibody (Invitrogen, A6455) was used. Sections were mounted with Dako-fluorescent mounting media (Dako North America) prior to imaging with an Olympus FV 1000 confocal microscopy.

\section{Fluorescence microangiography}

The fluorescein-coupled latex beads (Molecular Probes) were injected into the inflow tract of the atrium of the zebrafish embryo at $30 \mathrm{hpf}$ with a dose of $2 \mathrm{nl}$. The beads flowed with blood circulation and labeled all the vascular vessels of wild-type embryos in 5 minutes.

\section{Flow cytometry analysis}

Embryos at $28 \mathrm{hpf}$ were dissected in DPBS (Invitrogen) after dechorionation and deyolking [46], and digested with $1 \times$ trypsin/EDTA (Life Technologies) for $30 \mathrm{~min}$ at $28.5^{\circ} \mathrm{C}$. Single cell suspension was obtained by centrifugation at $400 \mathrm{~g}$ for $5 \mathrm{~min}$, washed twice with 0.9XPBS/
$5 \%$ FBS, and passed through a $40 \mu$ m nylon mesh filter. Fluorescence-activated cell sorting was performed with FACSAria (BD Biosciences) to quantify the frequency of the $\mathrm{GFP}^{+}$endothelial cells.

\section{Image analysis}

Embryos were imaged under an Olympus FV 1000 confocal microscopy equipped with the FV10-ASW version3 software.

\section{Statistical analysis}

The values were represented as mean \pm SEM. Two-tailed students't-tests were used for two groups comparison analysis, and $\mathrm{P}<0.05$ was considered to be significant. The bar charts were used to reflect the alterations of experimental data.

\section{Additional files}

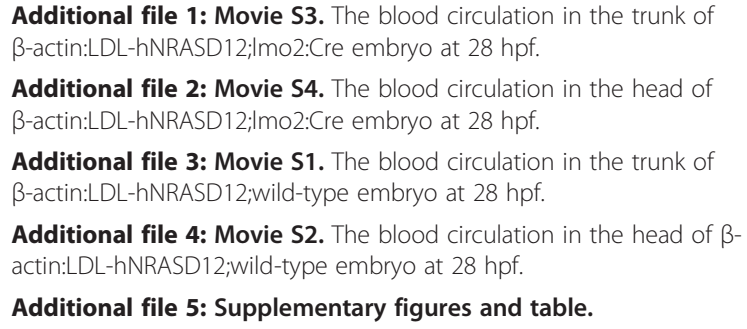

Additional file 2: Movie S4. The blood circulation in the head of $\beta$-actin:LDL-hNRASD12;Imo2:Cre embryo at $28 \mathrm{hpf}$.

Additional file 3: Movie S1. The blood circulation in the trunk of $\beta$-actin:LDL-hNRASD12;wild-type embryo at $28 \mathrm{hpf}$.

Additional file 4: Movie S2. The blood circulation in the head of $\beta$ actin:LDL-hNRASD12; wild-type embryo at $28 \mathrm{hpf}$.

Additional file 5: Supplementary figures and table.

\section{Competing interests}

The authors declare that they have no competing interests.

\section{Authors' contributions}

CGR performed experiments and analyzed data. LW, XEJ, YJL, ZWD, YZ(Yong Zhou), YJ, YC, and MD assisted with experiments. TXL, WJP, YZ(Yi Zhou), RBR, and CGR designed the research plan and wrote the paper. All authors were involved in the writing and final approval of the manuscript.

\section{Acknowledgments}

We thank Leonard Zon for providing zebrafish $\beta$-actin promoter plasmid; Brian Ciruna for providing the pDestTol2 vector and pCS-TP plasmids; Jiu-lin Du for providing the $T g(f l k 1$ :mCherry) fish; Qing Jing and Wen-Qing Li for the ephrinB2, notch3, hRT, flt4 and ephB4 riboprobes, and the vegfaa 121 and flk1PolyA-pBSKI2 plasmids; Tao Zhong for the grl riboprobe; All members of our laboratory for their technical assistance and helpful discussions. This work was supported in part by National Key Basic Research Program of China/973 Program (2013CB910900), National Natural Science Foundation of China (30525019, 30830047 and 30771185), Innovation Program of The Chinese Academy of Sciences (KSCX1-YW-R-03), E-Institutes of Shanghai Municipal Education Commission (E03003) and National Thousand Talents Program for Distinguished Young Scholars.

\section{Author details}

${ }^{1}$ Key Laboratory of Stem Cell Biology, Institute of Health Sciences, Shanghai Institutes for Biological Sciences, Chinese Academy of Sciences \& Shanghai Jiao Tong University School of Medicine, Shanghai, China. ${ }^{2}$ Shanghai Institute of Hematology, RuiJin Hospital, Shanghai Jiao Tong University School of Medicine, Shanghai, China. ${ }^{3}$ Stem Cell Program, Hematology/Oncology Program at Children's Hospital Boston, Harvard Medical School, Boston, MA 02114, USA. 
Received: 7 March 2013 Accepted: 4 May 2013

Published: 12 May 2013

\section{References}

1. Malumbres M, Barbacid M: RAS oncogenes: the first 30 years. Nat Rev Cancer 2003, 3:459-465.

2. Drosten M, Dhawahir A, Sum EY, Urosevic J, Lechuga CG, Esteban LM, Castellano E, Guerra C, Santos E, Barbacid M: Genetic analysis of Ras signalling pathways in cell proliferation, migration and survival. EMBO J 2010, 29:1091-1104.

3. Fernandez-Medarde A, Santos E: Ras in cancer and developmental diseases. Genes Cancer 2011, 2:344-358.

4. Parikh C, Subrahmanyam R, Ren R: Oncogenic NRAS rapidly and efficiently induces CMML- and AML-like diseases in mice. Blood 2006, 108:2349-2357.

5. Omidvar N, Kogan S, Beurlet S, le Pogam C, Janin A, West R, Noguera ME, Reboul M, Soulie A, Leboeuf $\mathrm{C}$, et al: BCL-2 and mutant NRAS interact physically and functionally in a mouse model of progressive myelodysplasia. Cancer Res 2007, 67:11657-11667.

6. Wang J, Liu Y, Li Z, Wang Z, Tan LX, Ryu MJ, Meline B, Du J, Young KH Ranheim $E$, et al: Endogenous oncogenic Nras mutation initiates hematopoietic malignancies in a dose- and cell type-dependent manner. Blood 2011, 118:368-379.

7. Li Q, Haigis KM, McDaniel A, Harding-Theobald E, Kogan SC, Akagi K Wong JC, Braun BS, Wolff L, Jacks T, Shannon K: Hematopoiesis and leukemogenesis in mice expressing oncogenic NrasG12D from the endogenous locus. Blood 2011, 117:2022-2032.

8. Kranenburg O, Gebbink MF, Voest EE: Stimulation of angiogenesis by Ras proteins. Biochim Biophys Acta 2004, 1654:23-37.

9. Eerola I, Boon LM, Mulliken JB, Burrows PE, Dompmartin A, Watanabe $S$, Vanwijck R, Vikkula M: Capillary malformation-arteriovenous malformation, a new clinical and genetic disorder caused by RASA1 mutations. Am J Hum Genet 2003, 73:1240-1249.

10. Wang HU, Chen ZF, Anderson DJ: Molecular distinction and angiogenic interaction between embryonic arteries and veins revealed by ephrin-B2 and its receptor Eph-B4. Cell 1998, 93:741-753.

11. Gault J, Sarin H, Awadallah NA, Shenkar R, Awad IA: Pathobiology of human cerebrovascular malformations: basic mechanisms and clinical relevance. Neurosurgery 2004, 55:1-16. discussion 16-17.

12. Krebs LT, Shutter JR, Tanigaki K, Honjo T, Stark KL, Gridley T: Haploinsufficient lethality and formation of arteriovenous malformations in Notch pathway mutants. Genes Dev 2004, 18:2469-2473.

13. Gambke C, Hall A, Moroni C: Activation of an N-ras gene in acute myeloblastic leukemia through somatic mutation in the first exon. Proc Natl Acad Sci U S A 1985, 82:879-882

14. Yamada Y, Pannell R, Forster A, Rabbitts TH: The oncogenic LIM-only transcription factor $\mathrm{Lmo} 2$ regulates angiogenesis but not vasculogenesis in mice. Proc Natl Acad Sci U S A 2000, 97:320-324.

15. Patterson LJ, Gering M, Eckfeldt CE, Green AR, Verfaillie CM, Ekker SC, Patient R: The transcription factors $\mathrm{Scl}$ and $\mathrm{Lmo} 2$ act together during development of the hemangioblast in zebrafish. Blood 2007, 109:2389-2398

16. Wang $L$, Zhang $Y$, Zhou T, Fu YF, Du TT, Jin $Y$, Chen $Y$, Ren $C G$, Peng $X L$, Deng M, Liu TX: Functional characterization of Lmo2-Cre transgenic zebrafish. Dev Dyn 2008, 237:2139-2146.

17. Zhou T, Wang L, Zhu KY, Dong M, Xu PF, Chen Y, Chen SJ, Chen Z, Deng M, Liu TX: Dominant-negative C/ebpalpha and polycomb group protein Bmi1 extend short-lived hematopoietic stem/progenitor cell life span and induce lethal dyserythropoiesis. Blood 2011, 118:3842-3852.

18. Orkin SH, Zon LI: Hematopoiesis: an evolving paradigm for stem cell biology. Cell 2008, 132:631-644.

19. Kissa K, Herbomel P: Blood stem cells emerge from aortic endothelium by a novel type of cell transition. Nature 2010, 464:112-115.

20. Bertrand JY, Chi NC, Santoso B, Teng S, Stainier DY, Traver D: Haematopoietic stem cells derive directly from aortic endothelium during development. Nature 2010, 464:108-111.

21. Paik EJ, Zon LI: Hematopoietic development in the zebrafish. Int J Dev Biol 2010, 54:1127-1137.
22. Bennett CM, Kanki JP, Rhodes J, Liu TX, Paw BH, Kieran MW, Langenau DM, Delahaye-Brown A, Zon LI, Fleming MD, Look AT: Myelopoiesis in the zebrafish, Danio rerio. Blood 2001, 98:643-651.

23. Gering $M$, Patient $R$ : Hedgehog signaling is required for adult blood stem cell formation in zebrafish embryos. Dev Cell 2005, 8:389-400.

24. North TE, Goessling W, Peeters M, Li P, Ceol C, Lord AM, Weber GJ, Harris J Cutting CC, Huang P, et al: Hematopoietic stem cell development is dependent on blood flow. Cell 2009, 137:736-748.

25. Jin SW, Beis D, Mitchell T, Chen JN, Stainier DY: Cellular and molecular analyses of vascular tube and lumen formation in zebrafish. Development 2005, 132:5199-5209.

26. Isogai S, Horiguchi M, Weinstein BM: The vascular anatomy of the developing zebrafish: an atlas of embryonic and early larval development. Dev Biol 2001, 230:278-301.

27. Fujita M, Cha YR, Pham VN, Sakurai A, Roman BL, Gutkind JS, Weinstein BM: Assembly and patterning of the vascular network of the vertebrate hindbrain. Development 2011, 138:1705-1715.

28. Cermenati S, Moleri S, Cimbro S, Corti P, Del Giacco L, Amodeo R, Dejana E, Koopman P, Cotelli F, Beltrame M: Sox 18 and Sox7 play redundant roles in vascular development. Blood 2008, 111:2657-2666.

29. Poole TJ, Coffin JD: Vasculogenesis and angiogenesis: two distinct morphogenetic mechanisms establish embryonic vascular pattern. J Exp Zool 1989, 251:224-231.

30. Herbert SP, Huisken J, Kim TN, Feldman ME, Houseman BT, Wang RA, Shokat KM, Stainier DY: Arterial-venous segregation by selective cell sprouting: an alternative mode of blood vessel formation. Science 2009, 326:294-298.

31. Parker LH, Schmidt M, Jin SW, Gray AM, Beis D, Pham T, Frantz G, Palmieri S, Hillan K, Stainier DY, et al: The endothelial-cell-derived secreted factor Egfl7 regulates vascular tube formation. Nature 2004 428:754-758.

32. Williams $\mathrm{C}$, Kim SH, Ni TT, Mitchell $L$, Ro $H$, Penn JS, Baldwin SH, Solnica-Krezel L, Zhong TP: Hedgehog signaling induces arteria endothelial cell formation by repressing venous cell fate. Dev Biol 2010, 341:196-204.

33. Swift MR, Weinstein BM: Arterial-venous specification during development. Circ Res 2009, 104:576-588.

34. Lawson ND, Mugford JW, Diamond BA, Weinstein BM: Phospholipase C gamma-1 is required downstream of vascular endothelial growth factor during arterial development. Genes Dev 2003, 17:1346-1351.

35. Lawson ND, Vogel AM, Weinstein BM: Sonic hedgehog and vascular endothelial growth factor act upstream of the Notch pathway during arterial endothelial differentiation. Dev Cell 2002, 3:127-136.

36. Westervelt P, Ley TJ: Seed versus soil: the importance of the target cell for transgenic models of human leukemias. Blood 1999, 93:2143-2148.

37. Renneville A, Roumier C, Biggio V, Nibourel O, Boissel N, Fenaux P Preudhomme C: Cooperating gene mutations in acute myeloid leukemia: a review of the literature. Leukemia 2008, 22:915-931.

38. Henkemeyer M, Rossi DJ, Holmyard DP, Puri MC, Mbamalu G, Harpal K Shih TS, Jacks T, Pawson T: Vascular system defects and neurona apoptosis in mice lacking ras GTPase-activating protein. Nature 1995 377:695-701.

39. Tuveson DA, Shaw AT, Willis NA, Silver DP, Jackson EL, Chang S, Mercer KL, Grochow R, Hock H, Crowley D, et al: Endogenous oncogenic K-ras(G12D) stimulates proliferation and widespread neoplastic and developmental defects. Cancer Cell 2004, 5:375-387.

40. Zon LI, Peterson RT: In vivo drug discovery in the zebrafish. Nat Rev Drug Discov 2005, 4:35-44.

41. Kimmel CB, Ballard WW, Kimmel SR, Ullmann B, Schilling TF: Stages of embryonic development of the zebrafish. Dev Dyn 1995, 203:253-310.

42. Kikuta $H$, Kawakami $K$ : Transient and stable transgenesis using tol2 transposon vectors. Methods Mol Biol 2009, 546:69-84

43. Borovina A, Superina S, Voskas D, Ciruna B: Vangl2 directs the posterior tilting and asymmetric localization of motile primary cilia. Nat Cell Bio 2010, 12:407-412.

44. Jing $C B$, Chen $Y$, Dong $M$, Peng $X L$, Jia XE, Gao L, Ma K, Deng M, Liu TX, Zon LI, et al: Phospholipase C gamma-1 is required for granulocyte maturation in zebrafish. Dev Biol 2013, 374:24-31. 
45. North TE, Goessling W, Walkley CR, Lengerke C, Kopani KR, Lord AM, Weber GJ, Bowman TV, Jang $\mathbb{H}$, Grosser T, et al: Prostaglandin E2 regulates vertebrate haematopoietic stem cell homeostasis. Nature 2007, 447:1007-1011.

46. Link V, Shevchenko A, Heisenberg CP: Proteomics of early zebrafish embryos. BMC Dev Biol 2006, 6:1.

doi:10.1186/1756-8722-6-34

Cite this article as: Ren et al:: Activated N-Ras signaling regulates arterial-venous specification in zebrafish. Journal of Hematology \& Oncology 2013 6:34.

\section{Submit your next manuscript to BioMed Central} and take full advantage of:

- Convenient online submission

- Thorough peer review

- No space constraints or color figure charges

- Immediate publication on acceptance

- Inclusion in PubMed, CAS, Scopus and Google Scholar

- Research which is freely available for redistribution 